\title{
Ultrasonographic Measurement of the Thickness of Axillary Recess Capsule in Healthy Volunteers
}

\author{
Kyoung Tae Kim, MD', Dong Gyu Lee, $\mathrm{MD}^{1}$, \\ Soyoung Lee, $\mathrm{MD}, \mathrm{PhD}^{1}$, Du Hwan Kim, $\mathrm{MD}^{1,2,3}$ \\ ${ }^{1}$ Department of Rehabilitation Medicine, ${ }^{2}$ Pain Research Center, and \\ ${ }^{3}$ Institute for Medical Science, Keimyung University School of Medicine, Daegu, Korea
}

Objective To evaluate the inter-rater and intra-rater reliability of ultrasonographic measurements of axillary recess (AR) thickness in healthy individuals, and to analyze the factors affecting the thickness of the AR capsule.

Methods We recruited 20 healthy individuals ( 10 male, 10 female) with a mean age of 37 years (standard deviation \pm 10 ). Two physiatrists (an experienced and a novice rater) independently investigated the AR thickness in three rounds. The AR thickness was measured for each individual at three shoulder abduction angles $\left(50^{\circ}, 70^{\circ}\right.$, and $\left.90^{\circ}\right)$. Intra-class correlation (ICC) coefficients were used to assess the reproducibility of each measurement.

Results Excellent intra-rater reliability coefficients were observed at the three shoulder abduction angles, in the analysis of both raters. The inter-rater reliability coefficient was also was excellent in both studies. There were significant differences in the AR thickness, according to the angle of shoulder abduction. The AR was thicker at $50^{\circ}$ than at $70^{\circ}$ and $90^{\circ}($ all $\mathrm{p}<0.001)$, and the AR was thicker at $70^{\circ}$ than at $90^{\circ}(\mathrm{p}<0.001)$. Height $(\mathrm{r}=0.62, \mathrm{p}=0.003)$ and body mass index $(r=0.52, p=0.019)$ were positively correlated with AR thickness. Males had a thicker AR capsule than females at all three angles (all $\mathrm{p}<0.001$ ).

Conclusion Ultrasonographic measurements of AR thickness in healthy individuals demonstrate excellent intra-rater and inter-rater reliability. AR thickness may depend on anthropometric variables and position of the shoulder.

Keywords Reliability and validity, Axillary recess, Ultrasonography

\section{INTRODUCTION}

Frozen shoulder (FS) is a common cause of shoulder pain. In addition to pain, it is characterized by a reduc- tion in the range of motion of the shoulder [1]. The diagnosis of FS is usually based on history taking, physical examination, and plain radiography. Although the exact pathogenesis of FS is still unclear, temporal evolution

Department of Rehabilitation Medicine, Keimyung University School of Medicine, 56 Dalseong-ro, Jung-gu, Daegu 41931, Korea. Tel: +82-53-250-7477, Fax: +82-53-250-7205, E-mail: ri-pheonix@hanmail.net

ORCID: Kyoung Tae Kim (http://orcid.org/0000-0001-9355-8326); Dong Gyu Lee (http://orcid.org/0000-0002-4787-4448); Soyoung Lee (http://orcid. org/0000-0002-4713-0713); Du Hwan Kim (http://orcid.org/0000-0002-9980-8549).

(c) This is an open-access article distributed under the terms of the Creative Commons Attribution Non-Commercial License (http://creativecommons.org/ licenses/by-nc/4.0) which permits unrestricted noncommercial use, distribution, and reproduction in any medium, provided the original work is properly cited. Copyright $\odot 2016$ by Korean Academy of Rehabilitation Medicine 
from inflammation, to fibrosis of the rotator interval (RI), anterior joint capsule, and axillary recess (AR) seems to be the main pathologic process [2]. Magnetic resonance imaging (MRI) has revealed various changes in patients with FS, such as thickening, signal changes, or enhancement of the RI, including the coracohumeral ligament, long biceps tendon, superior glenohumeral ligament, and AR [3-9]. The reliability of AR thickness measurements using MRI is high [10]. Few studies that assessed the diagnostic value of ultrasonography (US) in FS have been published, but the region of interest was the RI, and the results were controversial $[11,12]$. Recently, US examinations of glenohumeral synovitis of the aspect of the AR or posterior capsule have been included in the revised diagnostic criteria for polymyalgia rheumatica [13]. However, glenohumeral synovitis is usually investigated from the posterior capsule rather than AR [14]. There have been few reports of US examination of AR in normal subjects or patients with FS.

The objective of the current study was to test the interand intra-rater reliability of the US measurements of AR thickness in healthy volunteers, and to analyze the factors affecting the AR thickness. The results of the current study will expand the area of clinical applications of AR measurements in the diagnosis of FS.

\section{MATERIALS AND METHODS}

Right-handed healthy adult volunteers were recruited to participate in this study. We used numerical computation to optimize the design configuration. The sample size was calculated so as to detect a difference in the intra-class correlation (ICC) coefficients between 0.95 (exact reliability) and 0.85 (approximate reliability) at the $5 \%$ significance level, and with $80 \%$ power, assuming that two observers rated each participant three times. From this calculation, the minimal number of participants required was 20 [15]. The exclusion criteria for this study were as follows: (1) previous shoulder or neck pain (highenergy trauma of the shoulder or neck, symptomatic rotator cuff pathology, radicular pain in the interscapular area or upper limb, inflammatory arthropathy-related pain, or limitation of the range of shoulder motion); (2) previous history of shoulder or cervical spine surgery; (3) shoulder instability. Twenty individuals were included in this study (10 male, 10 female). Their mean age was 37 years (range, 21-52 years) and the mean body mass in$\operatorname{dex}$ (BMI) was $22.1 \mathrm{~kg} / \mathrm{m}^{2}$ (standard deviation \pm 2.2 ). This study protocol was approved by the regional Institutional Review Board (IRB Protocol No. 13-05).

\section{Procedure}

The AR thickness was measured using US in the supine position. The AR thickness was measured from both sides of the shoulder in each individual, at three shoulder abduction angles $\left(50^{\circ}, 70^{\circ}\right.$, and $\left.90^{\circ}\right)$ with neutral rotation (Fig. 1). The shoulder abduction angle is not equal to the angle of true glenohumeral joint abduction but is the angle of the global shoulder joint. Participants' elbow joints were flexed at $90^{\circ}$ and the forearms were neutral. US measurements were performed with Accuvix V10 machine (Samsung Medison, Seoul, Korea) with a 5-13 MHz linear transducer. The US probe was placed on the anterior pectoral line, which is located between the anterior axillary fold and coracobrachialis muscle, along the long axis of the humeral shaft (Fig. 1). The coronal view of T2weighted shoulder MRI ( $50^{\circ}$ abduction angle) showing the AR and a schematic illustration of AR were provided to the reader to enhance an understanding of the US measurements of AR thickness (Fig. 2). The frozen image that best visualized the cortical line of the humerus was selected to measure the AR thickness. The AR thickness was measured by the real-time method using the caliper on the ultrasound machine. It was determined as the distance from the bony cortex to the outer margin of the glenoid side capsule perpendicular to an imaginary midline between the tip of the humeral head and the surgical neck. Thus, the AR thickness is defined as the total summation of each thickness of the glenoid and humeral capsules (Fig. 3).

Two physiatrists (an experienced and a novice rater) measured the AR thickness sequentially at each shoulder abduction angle. The novice rater received 3 hours of training on AR anatomy, US measurements, and position of the subject, and practiced on another healthy volunteer to become familiar with the procedure before commencing the study. Two raters independently measured AR thickness in each participant three times with a time interval of 1 hour to blind to their previous own measurement and to let the participants to move out of the fixed position. The two raters were blinded to each other's measurements. 

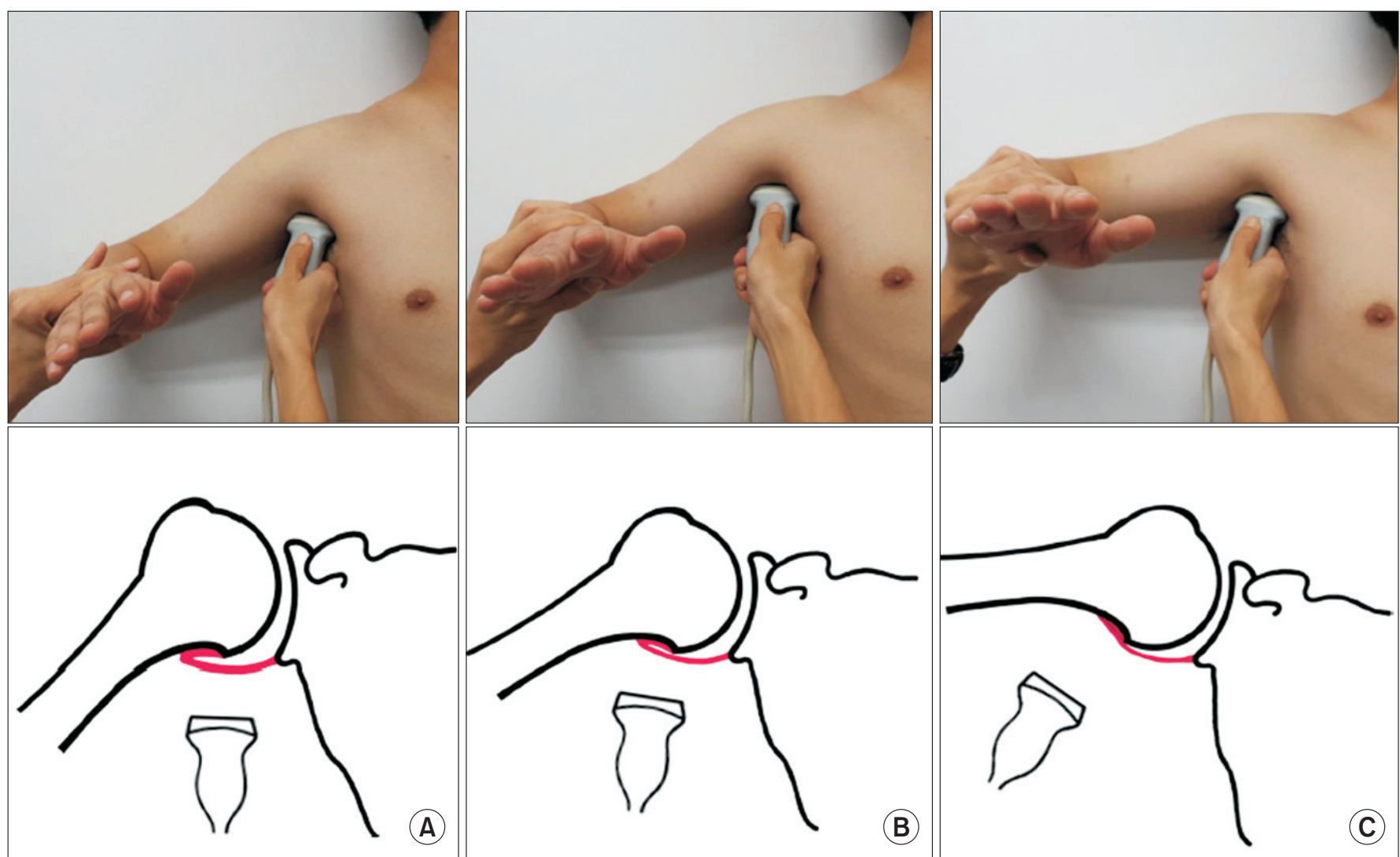

Fig. 1. Evaluation of the axillary recess capsule, using ultrasonography and schematic drawings, at three different positions according to the degrees of shoulder abduction $\left(50^{\circ}, 70^{\circ}\right.$, and $\left.90^{\circ}\right)$.
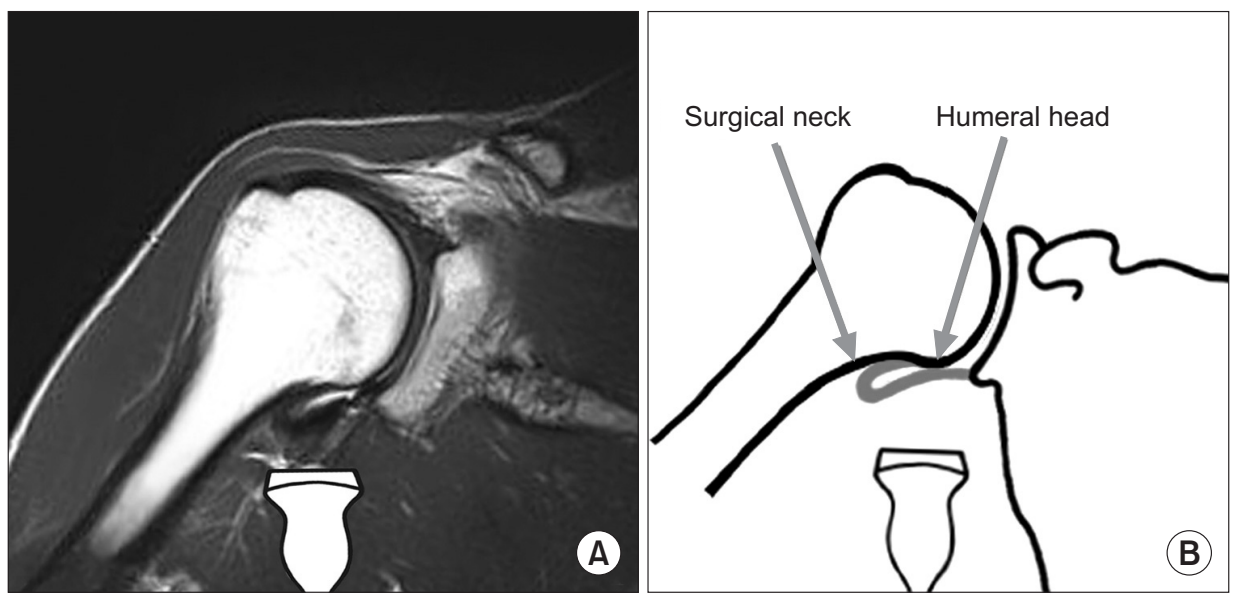

Fig. 2. (A) Coronal view of shoulder magnetic resonance imaging showing the region of interest in the ultrasonographic measurement of axillary recess thickness. (B) Schematic illustration of axillary recess.

\section{Data analysis}

To assess the inter-rater and intra-rater reliability of US measurements, the two-way random absolute agreement method of obtaining ICC with $95 \%$ confidence intervals was used. The ICC coefficients for AR thickness measurements between two raters for three trials $\left(\mathrm{ICC}_{2,2}\right)$ and across the three trials for each rater $\left(\mathrm{ICC}_{2,1}\right)$ were as- sessed. Paired t-test was used to examine the difference in AR thickness between left and right sides, and the differences in the average AR thickness at different angles of shoulder abduction. Student t-test was applied to compare the AR thickness in males and females. Pearson correlation was used to evaluate the relationship of AR thickness with height and BMI. Data were analyzed by 
using SPSS ver. 21.0 (IBM, Armonk, NY, USA), Microsoft Excel and statistical software R.

\section{RESULTS}

Descriptive data for the AR thickness for both raters at the three different angles, averaged across the three trials, is shown in Table 1. There were no significant differences between the left and right sides at any of the three angles (Table 1). The inter-rater reliability coefficient was 0.98 at right side and 0.96 at left side. The agreement between the experienced rater and the novice rater was excellent, according to the criteria of Shrout and Fleiss (excellent reliability $\geq 0.75$; fair to good reliability $0.40-0.74$; poor re-
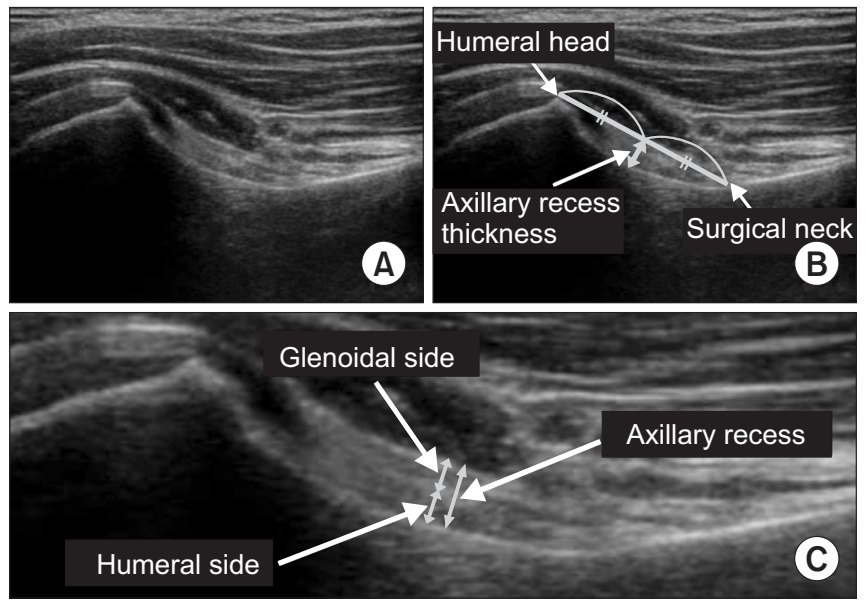

Fig. 3. (A) Axillary recess (AR) capsule on a frozen image. (B) Determination of the thickness of the AR capsule. (C) Humeral and glenoid sides of the AR capsule. The AR thickness is defined as the total summation of each thickness of the glenoid and humeral capsules. liability <0.40) [16]. The intra-rater reliability coefficients were $0.98,0.95$, and 0.96 at right side and $0.98,0.97$, and 0.96 at left side at each angle for the experienced rater, and $0.97,0.96$, and 0.95 at right side and 0.96, 0.96, and 0.95 at left side for the novice rater (Table 2). There were significant differences in the AR thickness according to the shoulder abduction angles (Fig. 4). The more the shoulder was abducted, the thinner the joint AR capsule was: the AR was thicker at $50^{\circ}$ than at $70^{\circ}$ and $90^{\circ}$ (all $\mathrm{p}<0.001$ ), and the AR was thicker at $70^{\circ}$ than at $90^{\circ}$ $(\mathrm{p}<0.001)$. Males had a thicker AR capsule than females $(3.22 \pm 0.52,2.89 \pm 0.41$, and $2.66 \pm 0.35 \mathrm{~mm}$ in males vs. $2.54 \pm 0.23,2.30 \pm 0.18$, and $2.06 \pm 0.14 \mathrm{~mm}$ in females at $50^{\circ}$, $70^{\circ}$ and $90^{\circ}$, respectively; all $\left.\mathrm{p}<0.001\right)$. The height $(\mathrm{r}=0.62$, $\mathrm{p}=0.003)$ and BMI $(\mathrm{r}=0.52, \mathrm{p}=0.019)$ were positively correlated with AR thickness.

\section{DISCUSSION}

In this study, we assessed inter- and intra-rater reliability of US measurements of the AR thickness in healthy subjects and found both parameters to be excellent. It is reassuring that even a relative beginner in diagnostic US showed excellent reliability in the measurements of AR thickness, which may depend on anthropometric variables and position of the shoulder.

In 1934, Codman wrote about FS, "This is a class of cases which I find it difficult to define..."; the diagnosis of FS is still challenging $[1,17]$. There are no definite diagnostic criteria for FS because this disease has a wide spectrum of clinical findings, and the invasive arthroscopic diagnosis of this relatively self-limiting disease is unethical. There is no consensus as to the necessary extent of range-

Table 1. The thickness of axillary recess recorded by the two raters, depending on three different angles of shoulder abduction

\begin{tabular}{lcccc}
\hline \multirow{2}{*}{ Raters } & \multirow{2}{*}{ Angle of shoulder abduction } & \multicolumn{3}{c}{ Thickness (mm) } \\
\cline { 2 - 4 } & & Right & Left & p-value $^{\text {a) }}$ \\
\hline Experienced & $50^{\circ}$ & $2.79 \pm 0.57$ & $2.90 \pm 0.66$ & 0.059 \\
& $70^{\circ}$ & $2.48 \pm 0.47$ & $2.57 \pm 0.56$ & 0.061 \\
Novice & $90^{\circ}$ & $2.23 \pm 0.43$ & $2.32 \pm 0.66$ & 0.219 \\
& $50^{\circ}$ & $2.78 \pm 0.56$ & $2.92 \pm 0.54$ & 0.074 \\
& $70^{\circ}$ & $2.55 \pm 0.47$ & $2.66 \pm 0.54$ & 0.063 \\
\hline
\end{tabular}

Values are presented as mean \pm standard deviation.

${ }^{\text {a) }}$ Paired t-test between right and left side. 
Table 2. Intra-rater and inter-rater reliability coefficients (ICC)

\begin{tabular}{|lccc}
\hline & \multirow{2}{*}{ Angles of shoulder abduction } & \multicolumn{2}{c}{ ICC (95\% confidential interval) } \\
\cline { 3 - 4 } & & Right & Left \\
\hline Intra-rater reliability & $50^{\circ}$ & $0.985(0.969-0.994)$ & $0.980(0.959-0.991)$ \\
\hline Experienced & $70^{\circ}$ & $0.957(0.910-0.982)$ & $0.975(0.947-0.989)$ \\
& $90^{\circ}$ & $0.964(0.927-0.984)$ & $0.964(0.926-0.985)$ \\
\hline Novice & $50^{\circ}$ & $0.975(0.948-0.989)$ & $0.969(0.934-0.987)$ \\
& $70^{\circ}$ & $0.963(0.925-0.984)$ & $0.965(0.927-0.985)$ \\
\hline & $90^{\circ}$ & $0.951(0.897-0.979)$ & $0.957(0.912-0.981)$ \\
\hline
\end{tabular}

Intra-rater reliability $\left(\mathrm{ICC}_{2,1}\right)$, two-way random effect, single measures, absolute agreement; inter-rater reliability $\left(\mathrm{ICC}_{2,2}\right)$, two-way random effect, average measures, absolute agreement.

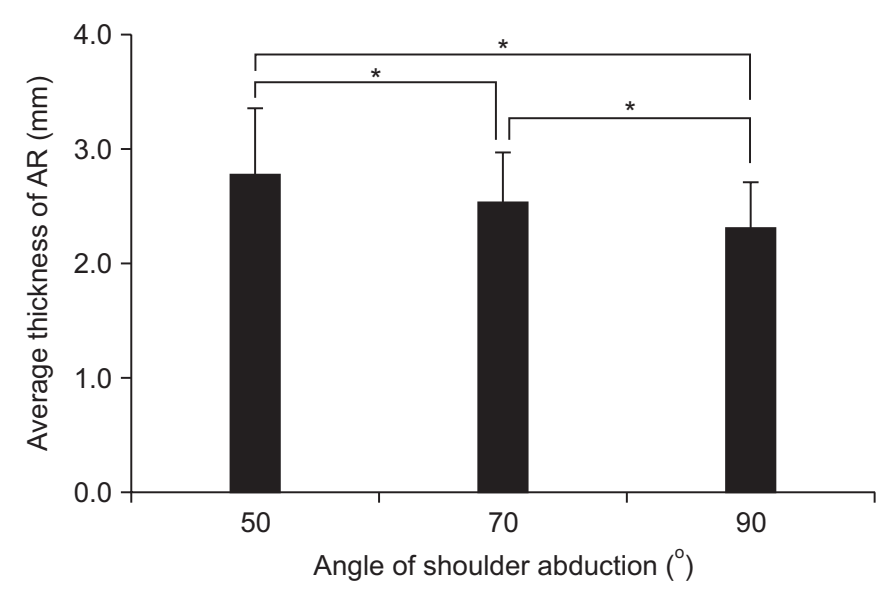

Fig. 4. Axillary recess (AR) thickness according to three different positions. ${ }^{*} \mathrm{p}<0.001$.

of-motion and imaging studies in the diagnosis of FS. In general, the diagnosis of FS is based on clinical findings and negative plain image. But painful shoulder stiffness can also be observed in other diseases like rotator cuff tear, calcific tendonitis, and rheumatic diseases $[1,2,18]$. Clinical diagnosis based solely on history taking and physical examination may lead to inappropriate treatment and delay the exact diagnosis. Recent research has demonstrated that imaging studies, including MRI, US, and positron emission tomography/computed tomography (PET/CT), have complementary roles in the diagnosis of FS [6-8,12,19-22].

Among imaging techniques, MRI is considered as the gold standard for diagnosis of shoulder pain. Many studies have demonstrated the presence of characteristic MRI features in FS, which could improve the diagnostic accuracy [3-7, 10,19,23-25]. Even though MRI has superior resolution, best visualization of deep soft tissue, and multiplanar sequencing, it is too expensive for this relatively benign disease. The advantages of US in comparison with MRI include better visualization of the rotator cuff, lower cost, faster examination, dynamic assessment, and ease of access [11]. Although US is one of the most useful tools in the diagnosis of shoulder diseases such as rotator cuff diseases, bursitis, and calcific tendinitis, its potential in the diagnosis of FS remains undetermined. Lee et al. [22] reported that combined examinations using power Doppler US and grey-scale echotexture in the RI yielded $87 \%$ sensitivity and $100 \%$ specificity in 30 patients with FS who had had symptoms for less than 1 year; in all patients, the diagnosis of FS was proven by arthroscopy. However, Walmsley et al. [12] demonstrated that only $29 \%$ of 41 participants with early-stage FS had increased vascularity in the RI. To date, the value of US findings in the RI for FS diagnosis remains controversial. In our opinion, hypoechoic echotexture and increased vascularity in the RI could be ambiguous and might be affected by the skill level of the examiner. We were interested in the AR capsule instead of the RI. To our knowledge, the US features of the AR capsule have not been reported. We thought that US measurements of the AR thickness should be validated and standardized before such measurements could be applied to the diagnosis of FS; these considerations prompted us to perform this study. The clinical value of our study is that it describes the US measurements of the AR thickness in healthy people for the first time. 
We measured the AR thickness as the total thickness of the glenoid and humeral capsules. Previous MRI studies have demonstrated heterogeneity in the measurements of the AR thickness. Lefevre-Colau et al. [10] measured the widest length, including glenoid and humeral side capsules, at its insertion in the humeral head perpendicular to the adjacent cortical bone. Jung et al. [19] measured the thickness of the glenoid and humeral capsules separately. On US, we could not reliably differentiate the glenoid capsule from the humeral capsule in a few participants. We thought that the total thickness of the glenoid and humeral capsules could be an appropriate measure for the thickness of the AR capsule for diagnostic purposes.

It is not surprising that the AR thickness was affected by the angle of shoulder abduction. We expected that it would be decreased by capsular stretching: the more the shoulder is abducted, the more stretched the joint capsule of the AR becomes. Some studies have demonstrated that the AR capsule may be elongated by the experimental hyperelastic model and recurrent anterior dislocation in clinical setting $[26,27]$. As a consequence, we hypothesized that AR thickness might be affected by the degree of shoulder abduction. The three specific angles $\left(50^{\circ}\right.$, $70^{\circ}$, and $90^{\circ}$ ) were arbitrarily chosen; the minimum angle was selected at $50^{\circ}$, considering the access of the ultrasound probe to the axillary area. Whereas the shoulder is adducted during MRI acquisition, it should inevitably be somewhat abducted during US when the probe approaches the axillary area. The position of the shoulder should be standardized during US measurement of AR thickness. We think $50^{\circ}$ as the most appropriate angle of shoulder abduction because some patients with FS cannot abduct their shoulders to $70^{\circ}$ or $90^{\circ}$. We also found that the height and BMI positively correlated with AR thickness. We surmise that this is because heavy-set people usually have larger shoulder joints, and the difference between sexes in the AR thickness might be related to the differences in anthropometric variables. But, this interpretation is confined only to healthy subjects. In frozen shoulder patients, AR thickness might be affected by its own pathologic state more than anthropometric variables.

Some obvious limitations of this study should be acknowledged. First, the US approach to the AR could not be used to measure the AR thickness in the neutral position of the shoulder, which would correspond to the AR thickness obtained from MRI, because shoulder abduction of at least $50^{\circ}$ was required for the US probe to approach the axillary area. In our study, the degree of shoulder abduction was not the one of the glenohumeral joint but the range of motion of the global shoulder joint. Although most patients with FS can abduct the shoulder at more than $50^{\circ}$, a few patients with severe FS may be unable to do so. The second limitation was the accuracy of fixed angles during measurements. Because US examination is a dynamic process, the position of the participants could change during examination, which would affect the results. However, this limitation did not seem to pose considerable problems because the time of examination at each angle was short. Third, the participants in this study were relatively young (mean age, 37 years) and lean (mean BMI, $22.1 \mathrm{~kg} / \mathrm{m}^{2}$ ). Lastly, we identified high inter- and intra-rater reliability of US measurement of the thickness of AR capsules only in healthy volunteers. Further prospective study is needed to be performed in frozen shoulder patients. Correlation between US and MRI must be identified.

In conclusion, the present study demonstrated that the inter- and intra-rater reliability of US measurements of the AR thickness was excellent. The AR thickness was affected by the angle of shoulder abduction: the more the shoulder was abducted, the thinner the AR capsule became. We also found that AR thickness positively correlated with the height and BMI.

\section{CONFLICT OF INTEREST}

No potential conflict of interest relevant to this article was reported.

\section{REFERENCES}

1. Hannafin JA, Chiaia TA. Adhesive capsulitis: a treatment approach. Clin Orthop Relat Res 2000;372:95109.

2. Hsu JE, Anakwenze OA, Warrender WJ, Abboud JA. Current review of adhesive capsulitis. J Shoulder Elbow Surg 2011;20:502-14.

3. Lee MH, Ahn JM, Muhle C, Kim SH, Park JS, Kim SH, et al. Adhesive capsulitis of the shoulder: diagnosis using magnetic resonance arthrography, with arthroscopic findings as the standard. J Comput Assist 
Tomogr 2003;27:901-6.

4. Manton GL, Schweitzer ME, Weishaupt D, Karasick D. Utility of MR arthrography in the diagnosis of adhesive capsulitis. Skeletal Radiol 2001;30:326-30.

5. Mengiardi B, Pfirrmann CW, Gerber C, Hodler J, Zanetti M. Frozen shoulder: MR arthrographic findings. Radiology 2004;233:486-92.

6. Sofka CM, Ciavarra GA, Hannafin JA, Cordasco FA, Potter HG. Magnetic resonance imaging of adhesive capsulitis: correlation with clinical staging. HSS J 2008;4:164-9.

7. Song KD, Kwon JW, Yoon YC, Choi SH. Indirect MR arthrographic findings of adhesive capsulitis. AJR Am J Roentgenol 2011;197:W1105-9.

8. Tamai K, Akutsu M, Yano Y. Primary frozen shoulder: brief review of pathology and imaging abnormalities. J Orthop Sci 2014;19:1-5.

9. Tamai K, Yamato M. Abnormal synovium in the frozen shoulder: a preliminary report with dynamic magnetic resonance imaging. J Shoulder Elbow Surg 1997;6: 534-43.

10. Lefevre-Colau MM, Drape JL, Fayad F, Rannou F, Diche T, Minvielle F, et al. Magnetic resonance imaging of shoulders with idiopathic adhesive capsulitis: reliability of measures. Eur Radiol 2005;15:2415-22.

11. Ryu KN, Lee SW, Rhee YG, Lim JH. Adhesive capsulitis of the shoulder joint: usefulness of dynamic sonography. J Ultrasound Med 1993;12:445-9.

12. Walmsley S, Osmotherly PG, Walker CJ, Rivett DA. Power Doppler ultrasonography in the early diagnosis of primary/idiopathic adhesive capsulitis: an exploratory study. J Manipulative Physiol Ther 2013;36:42835.

13. Dasgupta B, Cimmino MA, Kremers HM, Schmidt WA, Schirmer M, Salvarani C, et al. 2012 Provisional classification criteria for polymyalgia rheumatica: a European League Against Rheumatism/American College of Rheumatology collaborative initiative. Arthritis Rheum 2012;64:943-54.

14. Ruta S, Rosa J, Navarta DA, Saucedo C, Catoggio LJ, Monaco RG, et al. Ultrasound assessment of new onset bilateral painful shoulder in patients with polymyalgia rheumatica and rheumatoid arthritis. Clin Rheumatol 2012;31:1383-7.

15. Walter SD, Eliasziw M, Donner A. Sample size and optimal designs for reliability studies. Stat Med
1998;17:101-10.

16. Shrout PE, Fleiss JL. Intraclass correlations: uses in assessing rater reliability. Psychol Bull 1979;86:420-8.

17. Codman E. The shoulder: rupture of the supraspinatus tendon and other lesions in or about the subacromial bursa. Boston: T. Todd Company; 1934.

18. Sher JS, Uribe JW, Posada A, Murphy BJ, Zlatkin MB. Abnormal findings on magnetic resonance images of asymptomatic shoulders. J Bone Joint Surg Am 1995;77:10-5.

19. Jung JY, Jee WH, Chun HJ, Kim YS, Chung YG, Kim JM. Adhesive capsulitis of the shoulder: evaluation with MR arthrography. Eur Radiol 2006;16:791-6.

20. Kim du H, Sung DH, Ga HY, Choi JY. Metabolic patterns of the shoulder joint on (18)F-fluorodeoxyglucose positron emission tomography/computed tomography in adhesive capsulitis. Ann Nucl Med 2014;28:136-44.

21. Kim I, Yi JH, Lee J, Bae JH, Lim JK, Yoon JP, et al. Limited subacromial gliding of the supraspinatus tendon during dynamic ultrasonography can predict a decrease in capacity and MR arthrographic features of the shoulder joint. Eur Radiol 2012;22:2365-70.

22. Lee JC, Sykes C, Saifuddin A, Connell D. Adhesive capsulitis: sonographic changes in the rotator cuff interval with arthroscopic correlation. Skeletal Radiol 2005;34:522-7.

23. Carrillon Y, Noel E, Fantino O, Perrin-Fayolle O, TranMinh VA. Magnetic resonance imaging findings in idiopathic adhesive capsulitis of the shoulder. Rev Rhum Engl Ed 1999;66:201-6.

24. Connell D, Padmanabhan R, Buchbinder R. Adhesive capsulitis: role of MR imaging in differential diagnosis. Eur Radiol 2002;12:2100-6.

25. Emig EW, Schweitzer ME, Karasick D, Lubowitz J. Adhesive capsulitis of the shoulder: MR diagnosis. AJR Am J Roentgenol 1995;164:1457-9.

26. Rainis EJ, Maas SA, Henninger HB, McMahon PJ, Weiss JA, Debski RE. Material properties of the axillary pouch of the glenohumeral capsule: is isotropic material symmetry appropriate? J Biomech Eng 2009;131:031007.

27. Urayama M, Itoi E, Sashi R, Minagawa H, Sato K. Capsular elongation in shoulders with recurrent anterior dislocation: quantitative assessment with magnetic resonance arthrography. Am J Sports Med 2003;31:64-7. 